\title{
Impact of Gabapentin and Pregabalin on Neurological Outcome After Central Nervous System Injury
}

\author{
Caleb Morton, B.S. ${ }^{1}$, Fen-Lei Chang, M.D., Ph.D. ${ }^{1,2}$ \\ ${ }^{1}$ Indiana University School of Medicine, ${ }^{2}$ Parkview Health Neuroscience
}

\section{Background and Purpose:}

Injury to the central nervous system (CNS) is often detrimental to the health, functionality, and quality of life in both the short- and long-term. Injuries that fall under this umbrella include traumatic brain injury (TBI), traumatic spinal cord injury (TSCI), and stroke. These types of injuries vary in what initiates them, but their proposed mechanisms leading to cell dysfunction and death are strikingly similar. There has been pre-clinical and limited retrospective data supporting the idea that gabapentin and pregabalin both have neuroprotective qualities and may alleviate some of the sub-acute damage initiated by these different injuries. The purpose of this study is to determine whether patients taking either gabapentin or pregabalin at the time of their injury tend to have better outcomes than patients with similar injuries who were not taking either one of the two medications.

\section{Methods:}

This is a retrospective chart review analysis of 600 patients admitted to Parkview Hospitals from 2016-2019 for TBI, TSCI, or stroke. The outcomes of patients taking either gabapentin or pregabalin with one of the prior diagnoses will be compared to patients with the same diagnosis who were not taking either of the medications mentioned. Statistical analysis will be performed to evaluate if any significant difference exists between the outcomes at discharge of patients taking either medication versus patients who were not.

\section{Results:}

Results will be listed as comparisons between patients grouped by injury, and sub-grouped by medication usage. P-values will be included to show significance of comparisons.

\section{Conclusion and Potential Impact:}

The main impact of this study is to provide evidence and support leading to a potential method to improve outcomes in patients with CNS injuries. Secondary impacts are providing basis for development of a CNS injury registry and support for developing a unified CNS injury assessment scale to allow comparison of the treatments of different CNS injuries. 